Psychology of Language and Communication 2010, Vol. 14, No. 2

DOI: $10.2478 /$ v10057-010-0007-4

\author{
MACIEJ HAMAN \\ University of Warsaw
}

\title{
INTERNALLY-DRIVEN CHANGE AND FEATURE CORRESPONDENCE IN OBJECT REPRESENTATION: A KEY TO CHILDREN'S ESSENTIALISM?
}

\begin{abstract}
Two experiments were run to investigate how preschoolers use the pattern of an object's change as a cue to noticing correlations among the object's subsequent features. Four-year-old children were familiarized with either an internally or externally-driven transformation of an object, and tested for identification of an animation that did not match the familiar sequence of the object's features. In both experiments children in the internal-change group identified the incorrect sequence significantly more quickly than in the external-change condition. These results strongly suggest that perception of internally-driven transformation facilitates the formation of and/or access to a representation of correspondences between subsequent features of an object. The possible role of this mechanism in essentialist thinking is discussed at the end of the paper.
\end{abstract}

Key words: object representation, feature correlations, change perception, essentialism

Perception of dynamic events is a rich source of conceptually relevant information. For example, the pattern of motion allows us to determine an object's structure, infer goals, predict the outcome of between-object interaction, link an object's features into cohesive bundles, and trigger other kinds of abstract conceptual thinking. However, while most research has focused on motion, less is known about perception of an object's change. The research presented here is an attempt to fill this gap.

Change can be highly informative about an object and its category. Many objects keep specific tracks of change; they develop, grow, wear. In some cases these changes have evident external causes, in other cases they are internally-driven (e.g., developmental changes of living things). Perception and understanding of an object's change involves mechanisms to track the object's identity, and feature correlations and succession. The perceiver should be able to recognize an object

Address for correspondence: Maciej Haman, University of Warsaw, Faculty of Psychology, Stawki 5/7, 00-183 Warsaw, Poland. E-mail: meh@psych.uw.edu.pl 
as the same entity even after very radical transformation, and to understand how its early, sometimes hidden properties determine its final state. At the conceptual level such a mechanism is comprised in the "essentialist stance" (S. Gelman, 2003). The purpose of the present study is to investigate a possible perceptual mechanism that could facilitate this way of thinking.

Change detection seems to be an important cognitive mechanism of object cognition. For example, infants pay attention to object changes in dynamic physical events very early in development (Wang \& Baillargeon, 2007), as far as the changing feature is included in the child's causal model of an event. Surprisingly, up to now no one has studied children's perception of internally-driven object transformation, although there have been studies of the conceptualization of natural change and its origins by preschoolers at the level of verbal descriptions. A well-known study by Keil (1989) showed that although 5-year-olds are indeterminate whether an animal can turn into another animal by surgery, they would not accept cross-ontological transformations (e.g., from an artifact into an animal). Other studies show that for children, the initial state, origin, and internal structure are the best predictors of the outcome of natural change. In one of Gelman and Wellman's (1991) experiments $58 \%$ of 4-year-olds consistently predicted that a seed will grow into its "parent" kind, even if cultivated like another plant species.

The hypothesis tested here is that perception of an internally-driven change facilitates representation of correspondences between an object's features, especially those that characterize the initial state and become invisible during the change, and the most salient final-state features. If true, this could be a possible perceptual mechanism triggering some form of an essentialist scheme. In order to test whether preschoolers make use of such mechanisms, we have designed a set of simple animations of objects that undergo either internally or externally-driven change with salient, visible features at the start and at the end of the process. To avoid any intervention from category knowledge, the objects were unfamiliar to the children, relatively simple, and lacking any features that could suggest ontological kind (living, artificial, or inanimate-natural).

\section{Experiment 1}

\section{Method}

\section{Participants}

Forty-nine 4-year-old children participated (29 girls, 20 boys, mean age 4;7, range $3 ; 8-5 ; 7)$. Twenty-four of them were randomly assigned to the "internal change" condition, and 25 to the "external change" condition. They were recruited from 5 preschools in Warsaw and mostly belonged to urban middle class families. Written informed consent was obtained from all participants' parents before testing. 
Figure 1. Sample set of animations (selected frames) A-C - internally-driven change: A, B.1 - familiarization (Experiment 1), C, B.2 - familiarization (Experiment. 2), A, C - test (Experiment 1 and 2; "correct" choices: C for Experiment 1, A for Experiment 2), D - exemplar of animation for the externally-driven change condition

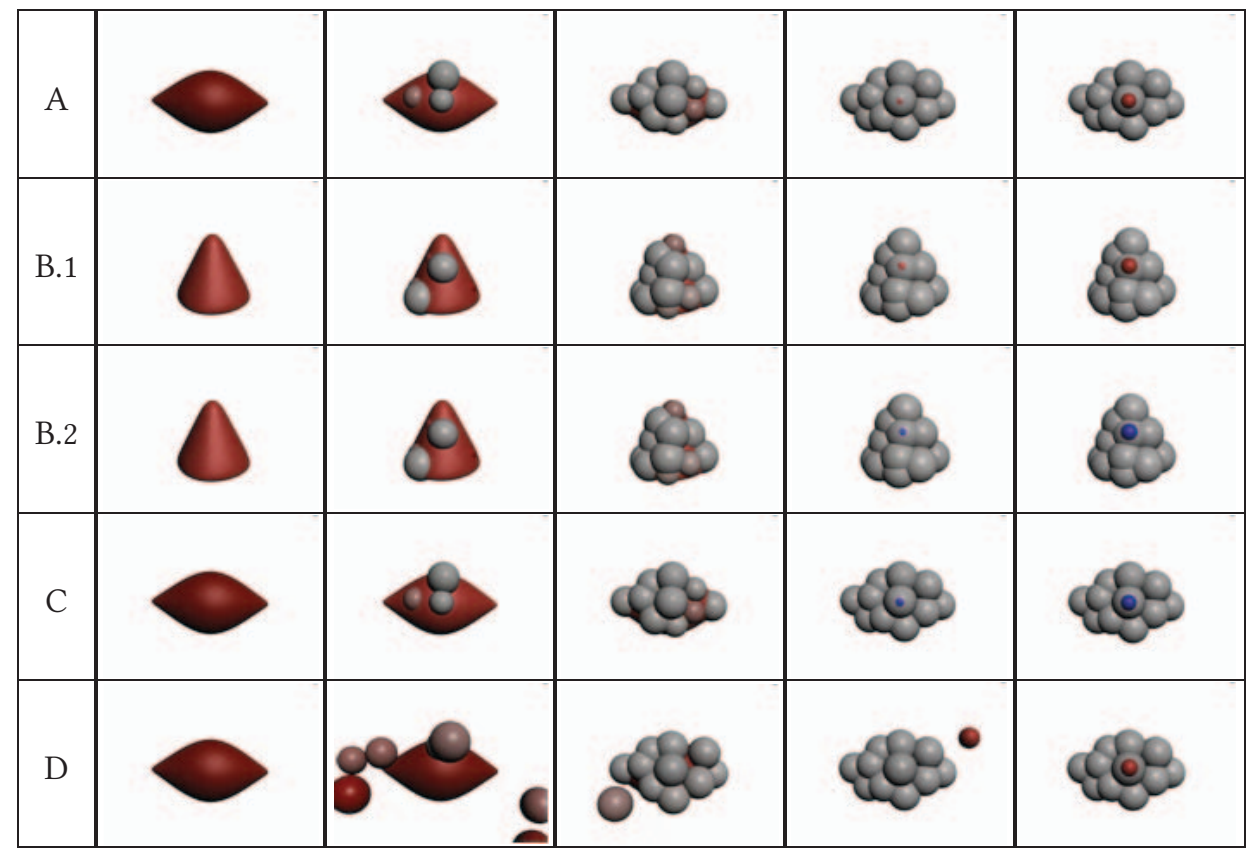

\section{Materials}

3D animations showing the transformation of one of two objects (a rounded cone-like one and a spindle-shaped one) were created. Each object could appear in one of two colors (red or blue). After 2 seconds of still phase a change started: spherical bubbles would either start growing from the object's surface (internallydriven change) or would start approaching from outside of the screen (external change). The bubbles initially matched the object's color, but they gradually turned gray. This phase lasted about 12 seconds, and as an outcome, the initial object was fully covered with gray bubbles. Then a smaller, colored (red or blue) bubble either grew from the object or flew in from outside, and got centrally placed on the transformed object. The whole event ended after 19.5 seconds from onset, and the image remained still for at least 2 seconds. A total of 16 animations were created ( 2 object shapes $\mathrm{x} 2$ colors $\mathrm{x} 2$ central bubble colors $\mathrm{x} 2$ patterns of change). Initial and final states for both internally and externally-driven change were identical. See Figure 1 for a sample set. 


\section{Procedure}

A PC-computer with a 17 " LCD touch-screen with integrated $1.5 \mathrm{~W}$ stereo speakers, run in 1280x1024 pixels resolution, was used. Children were tested in their schools in a separate room. First, they were taught how to use a touch-screen and accustomed to speaker-emitted instructions. After a simple introduction by the experimenter ("This is a special computer; you can touch the screen. It can also talk."), nine familiar objects (flowers, fruits, and simple artifacts) were displayed on the screen and the child was asked through speakers to touch one of them. Then the familiarization phase of the main procedure started. It consisted of four consecutive animations. The experimenter said "Now I will ask you for something else. I will show you four movies. Please watch them carefully but do nothing”. A 480x360 pixel window was opened randomly either on the left or right side of the screen, and the voice command "look!" was emitted. One second later, the first familiarization animation was displayed in the window. Depending on the condition, the animation was either of an internally or an externally-driven transformation. The initial color of the object and the color of the central bubble always matched (red-red or blue-blue). The second familiarization movie was displayed on the opposite side of the screen 2 seconds after the end of the first presentation, and it was also preceded by a "look!" command. The animation used in the second presentation involved a differently shaped object, but the color and change were preserved, so each child was familiarized for two shapes, but only one color scheme and one pattern of change. The next two familiarization trials were repetitions of the previous ones, except for presentation order and side (each of the two movies was shown once in the left and once in the right window).

The test phase began with the experimenter's instruction "Now I will show you two more movies. Watch them carefully. One of them is not perfect. At the end the computer will ask you to do something so touch the screen to do that". Then the command "look!" was emitted, and two windows were opened concurrently (one on the left and one on the right side). An animation was shown in each of the windows. One of these animations was the same as one of the two familiarization movies, whereas the second one differed from it only in that the color of the final, centrally located bubble was changed and therefore did not match the object's initial color (see Figure 1). One second after the object's transformation was completed, the command "show the imperfect one!" was emitted from the speakers, marking the onset of reaction-time measurement. The final state images were displayed in both windows until the child made a decision, or for 15 seconds from the end of the voice command. Reaction time and the choice were automatically recorded.

\section{Results}

The reaction was counted as "correct" if the child chose the movie with the new, not familiarized color scheme. Twelve children (83\%) in the internal-change condi- 
tion and 17 children (68\%) in the external-change condition made correct choices. The internal-change group performed significantly above chance, while the result of the external-change group was not decisive (one-tailed exact binomial $p<0.001$ and $p=0.053$ respectively). Three children in the internal-change and 7 children in the external-change condition made incorrect choices. One subject in each group did not choose within 15 seconds. However the between-group difference in the proportion of correct choices against incorrect and no-choice was not significant (Fisher exact $p=0.29$ ).

In order to test if internally-driven change facilitates access to representation of between-feature correspondence, we analyzed the reaction times of correct choices only. Mean group RTs for internal and external change (see Figure 2) were $4277 \mathrm{~ms}$ (2058), and $6155 \mathrm{~ms}$ (3709) respectively (standard deviations in parentheses). The difference was significant: $\mathrm{t}[24]=1.86, p<0.04^{1}$, one-tailed. Although it seems that children in both groups were (at least ultimately) able to code a correlation between the color of the initial object and the color of the central bubble at the end of the familiarization movies, nevertheless children in the internally-driven change group accessed this representation much more quickly.

\section{Experiment 2}

Experiment 1 showed that 4-year-olds presented with internally-driven change were able to access the representation of the correlation between initial and final state features much more quickly than those presented with an external-change condition. It is possible, however, that rather than using an abstract mechanism to deal with the feature succession, they relied on a more concrete one, such as "a red bubble should grow from the red object". Experiment 2 was designed to rule out this possibility. The procedure was the same as in experiment 1 , except that the color scheme in the familiarization phase was intersected (red central bubble rising from/flying onto the blue initial object and vice versa). Thus, the children's task was now to associate two different colors. If in experiment 1 the subjects simply expected that the bubble growing from the initial object would be of the same color, the internal change should not facilitate the subject's choices in the new design.

\section{Method}

Participants. Sixty children from 5 preschools in Warsaw participated (mean age 4;7, range 3;8-5;7, 29 female, 31 male). None of the subjects had participated in experiment 1 . Thirty children were randomly assigned to the internal-change group, and 30 to the external-change group. Written informed consent was obtained from all participants' parents before testing.

\footnotetext{
${ }^{1}$ Degrees of freedom and $t$ values are corrected for unequal variances in both experiments 1 and 2 . All other test assumptions, including normality, were satisfied.
} 
Figure 2. Mean correct reaction times for internally-driven and external change groups (Experiment 1 and Experiment 2)

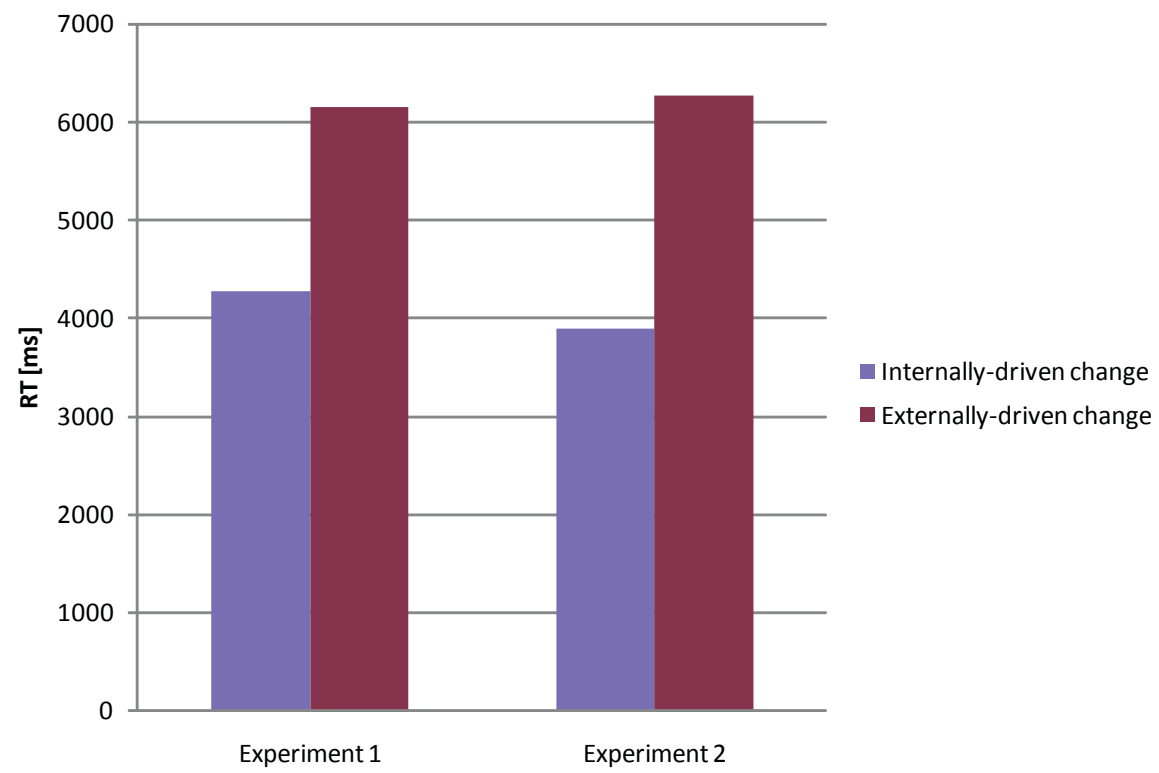

Materials and procedure. The same materials and procedure as in experiment 1 were used except that the animations presented in the familiarization phase exploited the intersected color scheme, i.e. animations starting with red initial objects ended with the blue central bubble and vice versa. The movie with matched colors was then the correct choice (non-fitting movie) in the test (see Figure 1).

\section{Results and discussion}

The results of experiment 2 almost exactly replicated those of experiment 1 , except that an insignificantly larger fraction of the internal-change group failed to choose correctly (Chi2[1] $=0.50, p=0.48$ for internal change, and Chi2[1] $=0.46$, $p=0.83$ for external change; actual frequencies tested against assumed equal proportions in both experiments). Twenty subjects (67\%) in the internally-driven change condition made correct choices, 7 (23\%) chose incorrectly, and $3(10 \%)$ did not choose in the 15-second time limit. Corresponding numbers for the externalchange group were: 19 (63\%), 8 (27\%), and $3(10 \%)$. Again the internal-change group performed above chance (exact binomial $p<0.05$ ), but the external-change group result was not significantly above chance level $(p=0.10)$ (again, the between-group difference was not significant, Fisher exact $p=0.99$, two-tailed). Group mean RTs for correct choices were $3887 \mathrm{~ms}(S D=1755)$ and $6271 \mathrm{~ms}(S D=3452)$ for the internally-driven and externally-driven change condition, respectively, and the 
difference was significant: $\mathrm{t}[26.21]=2.70, p<0.007$. RTs from both experiment 1 and 2 are shown in Figure 2.

We compared RTs of correct choices from experiments 1 and 2 in a 2(experiment)x2(pattern of change)x2(gender) ANOVA. Once again a strong effect of the pattern of change was found $(\mathrm{F}[1 ; 68]=10.06, p<0.003)$. No effects of experiment and gender or any interaction were found. A separate analysis also showed no effect or interaction of the specific set of animations used. Overall, the results clearly demonstrate that perception of internal change enhances integrated representation of two subsequent salient features of an object, even if the correspondence between features is arbitrary.

\section{General discussion and conclusion}

Children in both experiments made much quicker decisions based on feature correlations in the internally-driven than in the external-change condition. These results strongly suggest that internal change triggers a mechanism that facilitates the formation of and/or access to a representation of feature correspondences. Such a mechanism could be a part of at least the "sortal" and "placeholder" essentialist scheme (S. Gelman, 2003) that allows people to track an object's identity despite its change. Let us now speculate about the possible role of such a mechanism in conceptual development, and especially in the domain of naïve biology.

People seem to use "naïve theories" - sets of interrelated causal beliefs that determine important properties of objects and between-feature correlations (Murphy \& Medin, 1985). Dynamic events evoke this kind of mechanism at a very early stage of cognitive development in at least two core domains: in the "mechanics" of physical bodies and in understanding of intentions (see Baillargeon \& Wang, 2002; Gergely, Nádasdy, Csibra, \& Biro, 1995; Woodward, 1998, for only a few examples). It is less clear whether children are able to infer biological features from dynamic events (although they recognize biological motion from the first year of life, see Bertenthal, 1993). This is because animacy and intentionality are very closely related, especially from the child's perspective. From infancy to adulthood self-initiated, goal-directed motion is the strongest cue for life attribution (Opfer \& Siegler, 2004, R. Gelman, Durgin \& Kaufman, 1995, Scholl \& Tremoulet, 2000) and the structure of living objects (Rakison, 2005). At the same time, events involving self-initiated, goal-directed movement are subject to intentional explanation (Premack \& Premack, 1995; Scholl \& Tremoulet, 2000). Complex interrelations between biological and psychological explanations led some researchers (ranging from Piaget, 1929, to Carey, 1985) to skepticism about the independent conceptualization of biology in young children. On the other hand, there is a good deal of research showing that from preschool age onwards people expect living things to have specific and causally bound sets of features, which is a hallmark of theory-based thinking. These sets include substance features, color, internal and external parts, as well as behavior (R. 
Gelman et al., 1995; S. Gelman \& Wellman, 1991; Keil et al., 1998; Simons \& Keil, 1995). Some of the properties of an animate object determine its individual and kind identity, while other properties are more prone to change. Among the former are object origin and inner parts. The latter are surface properties. Among the reasons why people essentialize objects and categories is tracking of an object's individual (and kind) identity over sometimes radical transformations of surface form, size etc. While an object's visible features are not random and indeterminate, they are not necessarily constant, so the object's identity, or category, often cannot be derived from external properties only. Assuming that there is some internal property that initiates and controls the change, and determines surface properties, allows us to solve this problem. That does not necessarily imply knowledge of any specific causal mechanism - an "essence" as a "placeholder" for an unknown causal relation (or even only for an object kind) is enough for inductive reasoning about an object's features (Gelman, 2003). Cross-cultural and developmental studies show that this way of conceptualizing natural kinds is quite common at least from preschool age (Ahn et al., 2000; Atran, 1998; Inagaki \& Hatano, 2002; Tarlowski, 2006). Recently Newman, Herrmann, Wynn, and Keil (2008) showed that 18-month-old infants link the pattern of self-generated motion to an object's internal rather than external properties. However, no mechanism at the perceptual level, which promotes essentialist thinking, has been proposed as yet.

So far, however, we have clear evidence only for perceptual/attentional mechanisms of "on-line" feature binding, but not yet for a conceptual mechanism that could be built upon it. Some aspects require further research to clarify this issue. First, the animations used in the current study presented a continuous event that lasted less than 20 seconds. As opposed to this, natural biological change is slow, and it can hardly ever be observed as a continuous episode, but rather as a series of still images, often separated by a large time lag. Perhaps there is a special representational mechanism that integrates these still images into a "memory film", but the present study did not approach this problem. Another question is what aspect of internally-driven change facilitates the representation of feature correspondence. Is it, as we can expect from the essentialist hypothesis, an internal mechanism of change, or is it rather the object's cohesion during the entire episode that perhaps makes integrative work easier, either through initial principles of knowledge about a physical object (Spelke, 1990) or an attentional system for tracking objects and their representation in the form of object files (Kahneman, Treisman, \& Gibbs, 1992; Scholl, 2007)? Although the results of experiment 2 provide some indirect support for the internal mechanism explanation, more direct tests could be required. In a separate study (Haman, 2009) we have shown that the initial lack of an object's cohesion in the external-change condition does not lead to longer processing time if there are no salient features to be bound. Another test could consist of a comparison between two kinds of cohesive change: one with no visible cause, and one triggered by external intervention (e.g., a bar touching the object before the 
bubble's growth). Also, if the mechanism under consideration is expected to support essentialist thinking, it would be important to show that it not only supports an object's identification, but also allows transfer of the learned feature sequence to other objects that form a category. We also have some preliminary evidence for this (Haman, 2009), even in 14-month-old infants (Hernik \& Haman, 2010).

An extensive research program has just been designed to deal with the considerations listed above. Nevertheless the present study is the first demonstration that for children, the pattern of an object's change is an important factor in detecting feature correlations and that this, in turn, could be a crucial part of theory-driven essentialist categorization. This mechanism, although not necessarily limited to living kinds, could be especially useful for the development of biological concepts alternative to motion- and intentionality-based ones.

Extensive research by S. Gelman and her collaborators (Gelman, 1997, 2003; Gelman et al., 1998) has documented the role of linguistic cues in the activation of essentialist beliefs. Appropriate syntactic form and pragmatic context (e.g., generic statements in parents' speech directed to preschoolers) led to the formation of kindbased rather than episodic representation. Our study suggests that such linguistic and social mechanisms are also supported by some basic perceptual principles. Nevertheless a lot of research is necessary to explicitly show a link between these two levels in concept acquisition.

\section{Acknowledgments}

Research and preparation of this manuscript has been supported to the author by Ministry od Science and Higher Education grants for years 2006-2009 and 20092012. Author thanks Małgorzata Hawełko for designing animations for the study, and all students involved in testing children. Special thanks for all children, teachers, and parents from the preschools in which the study was run.

\section{References}

Ahn, W., Gelman, S.A., Amsterlaw, J.A., Hohenstein, J., \& Kalish, C.W. (2000). Causal status effect in children's categorization. Cognition, 76, B35-B43.

Atran, S. (1998). Folk biology and the anthropology of science. Behavioral and Brain Sciences, 21, 547-611.

Baillargeon, R. \& Wang, S. (2002). Event categorization in infancy. Trends in Cognitive Sciences, 6, 85-93.

Bertenthal B.I. (1993). Perception of biomechanical motions by infants: intrinsic image and knowledge-based constraints. In C. Granrud (Ed.), Carnegie Symposium on Cognition: Visual Perception and Cognition in Infancy (pp. 175-214). Hillsdale. NJ: Erlbaum.

Carey, S. (1985). Conceptual Change in Childhood. Cambridge, MA: MIT Press. 
Gelman, R., Durgin, F., \& Kaufman, L. (1995). Distinguishing between animates and inanimates: Not by motion alone. In D. Sperber, D. Premack, \& A. Premack (Eds.), Causal cognition: A multidisciplinary debate (pp. 150-184). Oxford: Clarendon Press.

Gelman, S.A. (1997). What does word use tell us about conceptual content? Psychology of Language and Communication, 1 (2), 5-16.

Gelman, S.A. (2003). The essential child: Origins of essentialism in everyday thought. Oxford: Oxford University Press.

Gelman, S.A., Coley, J.D., Rosengren, K., Hartman, E., \& Pappas, T. (1998). Beyond labeling: The role of parental input in the acquisition of richly structured categories. Monographs of the Society for Research in Child Development. Serial No. 253, Vol. 63, No. 1.

Gelman, S.A., \& Wellman, H.M. (1991) Insides and essences: Early understandings of the nonobvious. Cognition, 38, 213-244.

Gergely, G., Nádasdy, Z., Csibra, G., \& Bíró, S. (1995) Taking the intentional stance at 12 months of age. Cognition, 56, 165-193.

Hernik, M. \& Haman, M. (2010, January). Fourteen-month-olds transfer sequences of features derived from internally-driven object transformation. Cognitive Development Center Opening Conference, Central European University, Budapest.

Haman, M. (2009, June). Internally-driven object transformation enhances essentialist categorization. International Conference on Biological Understanding and Theory of Mind. Reims, France.

Inagaki, K. \& Hatano, G. (2002). Young Children's Thinking About the Biological World. New York: Psychology Press.

Keil, F.C. (1989). Concepts, kinds, and cognitive development. Cambridge, MA: The MIT Press.

Keil, F.C., Smith, W.C., Simons, D.J., \& Levin, D.T. (1998). Two dogmas of conceptual empiricism: Implications for hybrid models of the structure of knowledge, Cognition, 65, 103-135.

Kahneman, D., Treisman, A., \& Gibbs, B.J. (1992). The reviewing of object files: Object-specific integration of information. Cognitive Psychology, 24, 174-219.

Murphy, G.L., \& Medin, D.L. (1985). The role of theories in conceptual coherence. Psychological Review, 92, 289-316.

Newman, G.E., Herrmann P., Wynn K., \& Keil F.C. (2008). Biases towards internal features in infants'reasoning about objects. Cognition, 107, 420-432.

Opfer, J.E. \& Siegler, R.S. (2004). Revisiting preschoolers living things concept: A microgenetic analysis of conceptual change in basic biology. Cognitive Psychology, 49, 301-332.

Piaget, J. (1929). The child's conception of the world. New York: Harcourt.

Premack, D. \& Premack A.J. (1995). Intention as psychological cause. In D. Sperber, D. Premack, \& A.J. Premack (Eds.), Causal cognition: A multidisciplinary debate (pp. 185-199). New York, NY: Oxford University Press. 
Rakison, D.H. (2005). Developing knowledge of objects' motion properties in infancy Cognition, 96, 183-214.

Simons, D.J. \& Keil, F.C. (1995). An abstract to concrete shift in the development of biological thought: The insides story. Cognition, 56, 129-163.

Scholl, B.J. \& Tremoulet, P.D. (2000). Perceptual causality and animacy. Trends In Cognitive Sciences, 4, 299-309.

Scholl, B.J. (2007). Object Persistence in Philosophy and Psychology. Mind \& Language, 22, 563-591.

Spelke, E.S. (1990). Principles of object perception. Cognitive Science, 14, 29-56.

Tarlowski, A. (2006). If it's an animal it has axons: Experience and culture in preschool children's reasoning about animates. Cognitive Development, 21, 249-265.

Wang, S. \& Baillargeon, R. (2007). Detecting impossible changes in infancy: A threesystem account. Trends in Cognitive Sciences, 12, 17-23.

Woodward, A. (1998). Infants selectively encode the goal object of an actor's reach. Cognition, 69, 1-34. 